\title{
FUNCTIONAL LARGE DEVIATIONS AND MODERATE DEVIATIONS FOR MARKOV-MODULATED RISK MODELS WITH REINSURANCE
}

\author{
FUQING GAO*** AND \\ JUN YAN, ${ }^{* * * *}$ Wuhan University
}

\begin{abstract}
We establish a functional large deviation principle and a functional moderate deviation principle for Markov-modulated risk models with reinsurance by constructing an exponential martingale approach. Lundberg's estimate of the ruin time is also presented.
\end{abstract}

Keywords: Markov-modulated risk model; large deviations; moderate deviations; Lundberg's estimate

2000 Mathematics Subject Classification: Primary 60F10; 60G55; 60J27

Secondary $91 \mathrm{~B} 30$

\section{Introduction}

\subsection{Markov-modulated risk model}

Firstly, let us introduce the Markov-modulated risk model (cf. [13]). A process $\{N(t), t \geq 0\}$ is said to be a point process if

$$
N(t)=\sum_{l=1}^{\infty} \mathbf{1}_{\left\{T_{l} \leq t\right\}},
$$

where $\left\{T_{l}, l \geq 1\right\}$ is a sequence of stopping times such that $T_{1}>0$ almost surely (a.s.), $T_{l}<T_{l+1}$ on $\left\{T_{l}<\infty\right\}$ for any $l \geq 1$, and $\lim _{l \rightarrow \infty} T_{l}=\infty$ a.s. A point process $\{N(t), t \geq 0\}$ is said to be a Markov-modulated Poisson process if it is a doubly stochastic Poisson process with intensity $\lambda_{J(t)}$, where $J=\{J(t), t \geq 0\}$ is an irreducible continuous-time Markov chain with finite state space $E$ and the $\lambda_{i}, i \in E$, are positive numbers, i.e. the conditional characteristic function of $\{N(t), t \geq 0\}$ has the following expression:

$$
\mathrm{E}\left(\exp \{\mathrm{i} \theta(N(t)-N(s))\} \mid \mathcal{F}_{S}\right)=\exp \left\{\left(\mathrm{e}^{\mathrm{i} \theta}-1\right) \int_{s}^{t} \lambda_{J(u)} \mathrm{d} u\right\},
$$

where $\mathcal{F}_{s}=\sigma(N(u), u \leq s) \vee \sigma(J(u), u \geq 0)$.

Let $\pi_{i}, i \in E$, denote the stationary distribution of the Markov chain $J$.

Let $\left\{U_{l}, l \geq 1\right\}$ be a sequence of positive random variables, and let $G_{i}, i \in E$, be probability distributions with supports in $[0,+\infty)$. Assume that, for all $i \in E, \mu_{i}:=\int_{0}^{\infty} x G_{i}(\mathrm{~d} x)<\infty$

Received 4 April 2008; revision received 21 June 2008.

* Postal address: School of Mathematics and Statistics, Wuhan University, Wuhan 430072, P. R. China.

This work was supported by the National Natural Science Foundation of China (grant number 10571139).

** Email address: fqgao@whu.edu.cn

*** Email address: yanjun@mail.whu.edu.cn 
and that

- $U_{l}, l \geq 1$, and $\{N(t), t \geq 0\}$ are conditionally independent given $J$,

- for each $l \geq 1$, the conditional distribution of $U_{l}$ given $J$ is $G_{J\left(T_{l}\right)}$.

A reinsurance policy is a measurable function from $\mathcal{R}:[0, \infty) \times[0, \infty) \rightarrow[0, \infty)$ which satisfies $0 \leq R_{t}(\alpha) \leq \alpha$, where $R_{t}(\alpha)=\mathcal{R}(t, \alpha)$. The condition $0 \leq R_{t}(\alpha) \leq \alpha$ represents the part of the claim that the company pays when a claim of size $\alpha$ occurs at time $t$.

A Markov-modulated risk process with a reinsurance policy $\mathcal{R}$ is defined by

$$
X_{\mathcal{R}}^{x}(t)=x+p_{\mathcal{R}}(t)-S_{\mathcal{R}}(t),
$$

where $x>0$ is the initial capital; $p_{\mathcal{R}}(t)=p t-q_{\mathcal{R}}(t)$ is the deterministic premium, $p$ is a constant premium rate which has the form

$$
p=(1+\kappa) \sum_{i \in E} \pi_{i} \lambda_{i} \mu_{i}
$$

for some relative safety loading $\kappa>0$, and $q_{\mathcal{R}}(t)$ is the premium up to time $t$ paid by the insurer to the reinsurer which has the form

$$
q_{\mathcal{R}}(t)=(1+\eta) \sum_{i \in E} \pi_{i} \lambda_{i} \int_{0}^{t}\left(\mu_{i}-\int_{0}^{\infty} R_{S}(x) G_{i}(\mathrm{~d} x)\right) \mathrm{d} s
$$

for some relative safety loading $\eta>0$; and

$$
S_{\mathcal{R}}(t)=\sum_{l=1}^{N(t)} R_{T_{l}}\left(U_{l}\right)
$$

is the aggregate claims process, $\left\{R_{T_{l}}\left(U_{l}\right), l \geq 1\right\}$ is the sequence of claims, $N(t)$ is the claims number process, which is a doubly stochastic Poisson process with intensity $\lambda_{J(t)}$, and $J=$ $\{J(t), t \geq 0\}$ is an irreducible continuous-time Markov chain with finite state space $E$.

The Markov-modulated risk process with reinsurance is a generalization of the classical case. For example, if $G_{i}=G$ and $\lambda_{i}=\lambda$ for all $i \in E$, and $\left\{U_{l}, l \geq 1\right\},\{N(t), t \geq 0\}$, and $J$ are independent, then $S_{\mathcal{R}}(t)$ is the classic case. Recently, Macci and Stabile [13] studied the large deviations and ruin probability of the Markov-modulated risk process with reinsurance and obtained a functional large deviation principle for the classic case. Large deviations of some risk processes and applications of large deviations to insurance have received much attention in the research literature; see, for example, [1, p. 306], [2], [6], [9, p. 85], [12], [13], and [14]. In this paper we present an exponential martingale method to establish large deviations and moderate deviations for risk processes, and obtain the functional large deviation principle, the functional moderate deviation principle, and Lundberg's estimate of the ruin time for the Markov-modulated risk process with reinsurance.

The rest of the paper is organized as follows. In Subsection 1.2 we introduce some large deviations terminology, the Gärtner-Ellis theorem, and a result on functional large deviations used in this paper. In Section 2 we construct an exponential martingale associated with the Markov-modulated risk model which plays an important role in this paper. The functional large deviation principle is established in Section 3. The moderate deviations are studied in Section 4. In Section 5 we give an estimate for the ruin probability (Lundberg's estimate) using the exponential martingale method. 


\subsection{Preliminaries}

In this subsection we introduce large deviations (cf. [4, Chapter 2] and [5, Chapter 4]). Let $S$ be a metric space with metric $d$, and let $\left\{Y_{\alpha}: \alpha>0\right\}$ be a family of $S$-valued random variables. Denote the law of $Y_{\alpha}$ by $\mu_{\alpha}$. Let $\lambda(\alpha)$ be a sequence of positive real numbers satisfying $\lambda(\alpha) \rightarrow \infty$ as $\alpha \rightarrow \infty$.

(i) A function $I(\cdot): S \rightarrow[0,+\infty]$ is said to be a rate function if it is lower semicontinuous and it is said to be a good rate function if its level set $\{x \in S: I(x) \leq a\}$ is compact for all $a \geq 0$.

(ii) The family of probability measures $\left\{\mu_{\alpha}: \alpha>0\right\}$ (or the family $\left\{Y_{\alpha}: \alpha>0\right\}$ ) is said to satisfy a large deviation principle (LDP) with speed $\lambda(\alpha)$ and rate function $I(\cdot)$ if, for any closed set $F$ and open set $G$ in $S$,

$$
\limsup _{\alpha \rightarrow \infty} \frac{1}{\lambda(\alpha)} \log \mu_{\alpha}\{F\} \leq-\inf _{x \in F} I(x), \quad \liminf _{\alpha \rightarrow \infty} \frac{1}{\lambda(\alpha)} \log \mu_{\alpha}\{G\} \geq-\inf _{x \in G} I(x) .
$$

In short form, we say that $\left(\mu_{\alpha}, I(\cdot), 1 / \lambda(\alpha)\right)$ satisfies an LDP.

(iii) The family $\left\{\mu_{\alpha}: \alpha>0\right\}$ is exponentially tight with speed $\lambda(\alpha)$ if, for every $L>0$, there is a compact set $K_{L}$ in $S$ such that

$$
\limsup _{\alpha \rightarrow \infty} \frac{1}{\lambda(\alpha)} \log \mu_{\alpha}\left(K_{L}^{\mathrm{c}}\right) \leq-L .
$$

Lemma 1.1. (Gärtner-Ellis theorem.) Let $\left\{Y_{\alpha}, \alpha>0\right\}$ be a family of random variables taking values in $\mathbb{R}^{d}$. Suppose that, for any $y \in \mathbb{R}^{d}$,

$$
\Lambda(y):=\lim _{\alpha \rightarrow \infty} \frac{1}{\lambda(\alpha)} \log \mathrm{E}\left(\exp \left\{\lambda(\alpha)\left\langle Y_{\alpha}, y\right\rangle\right\}\right) \in(-\infty,+\infty]
$$

exists and that $\Lambda(\cdot)$ is finite in a neighborhood of 0 , where $\langle x, y\rangle=\sum_{i=1}^{d} x_{i} y_{i}$ for $x, y \in \mathbb{R}^{d}$. If $\Lambda$ is essentially smooth then $\left\{Y_{\alpha}, \alpha>0\right\}$ satisfies the LDP with speed $\lambda(\alpha)$ and rate function $\Lambda^{*}$ defined by

$$
\Lambda^{*}(x)=\sup _{y \in \mathbb{R}^{d}}\{\langle x, y\rangle-\Lambda(y)\} .
$$

In particular, if $\Lambda$ is finite and Gäteaux differentiable then $\left\{Y_{\alpha}, \alpha>0\right\}$ satisfies the LDP with speed $\lambda(\alpha)$ and rate function $\Lambda^{*}$.

Let $D([0,1])$ be the space of càdlàg functions (i.e. those which are right continuous with left limits) from $[0,1]$ to $\mathbb{R}$ equipped with the uniform metric $d(x, y):=\sup _{t \in[0,1]}|x(t)-y(t)|$.

Lemma 1.2. (cf. [7].) Let $\left\{\mu_{\alpha}, \alpha>0\right\}$ be a family of probability measures on $D([0,1])$. For any finite subset $\left\{t_{1}, \ldots, t_{l}\right\} \subset[0,1]$, set $\mu_{\alpha}^{t_{1}, \ldots, t_{l}}=\mu_{\alpha} \circ \pi_{t_{1}, \ldots, t_{l}}^{-1}$, where $\pi_{t_{1}, \ldots, t_{l}}: x \rightarrow$ $\left(x_{t_{1}}, \ldots, x_{t_{l}}\right)$ denotes the projection from $D([0,1])$ to $\mathbb{R}^{l}$. If, for any finite subset $\left\{t_{1}, \ldots, t_{l}\right\} \subset$ $[0,1],\left\{\mu_{\alpha}^{t_{1}, \ldots, t_{l}}, \alpha>0\right\}$ satisfies the LDP with speed $\lambda(\alpha)$ and rate function $I_{t_{1}, \ldots, t_{l}}\left(x_{1}, \ldots, x_{l}\right)$ in $\mathbb{R}^{l}$ and for any $\delta>0$,

$$
\lim _{\varepsilon \rightarrow 0} \sup _{s \in[0,1]} \limsup _{\alpha \rightarrow \infty} \frac{1}{\lambda(\alpha)} \log \mu_{\alpha}\left(\sup _{s \leq t \leq s+\varepsilon}|x(t)-x(s)| \geq \delta\right)=-\infty,
$$

then $\left\{\mu_{\alpha}, \alpha>0\right\}$ satisfies the LDP on $D([0,1])$ with speed $\lambda(\alpha)$ and good rate function defined by

$$
I(x):=\sup _{\left\{t_{1}, \ldots, t_{l}\right\} \subset[0,1]} I_{t_{1}, \ldots, t_{l}}\left(x_{t_{1}}, \ldots, x_{t_{l}}\right) .
$$




\section{Exponential martingale and Laplace functional}

The main purpose of this section is to construct an exponential martingale associated with the Markov-modulated risk model with reinsurance and to calculate the Laplace functional of the model.

Theorem 2.1. (i) Set

$$
M_{t}:=S_{\mathcal{R}}(t)-\int_{0}^{t} \int_{0}^{\infty} R_{u}(x) G_{J(u)}(\mathrm{d} x) \lambda_{J(u)} \mathrm{d} u .
$$

Then $\left\{M_{t}, g_{t}, t \geq 0\right\}$ is a martingale, where $g_{s}=\sigma(N(u), u \leq s) \vee \sigma(J(u), u \geq 0) \vee$ $\sigma\left(U_{l}, l \leq N(s)\right)$.

(ii) If, for some $\delta>0$,

$$
\sup _{i \in E} \int_{0}^{\infty} \mathrm{e}^{\delta x} G_{i}(\mathrm{~d} x)<\infty
$$

then, for any measurable function $\theta(t)$ satisfying $\sup _{t \geq 0} \theta(t)<\delta$,

$$
Z_{t}^{\theta}:=\exp \left\{\int_{0}^{t} \theta(u) \mathrm{d} S_{\mathcal{R}}(u)-\int_{0}^{t} \int_{0}^{\infty}\left(\exp \left\{\theta(u) R_{u}(x)\right\}-1\right) G_{J(u)}(\mathrm{d} x) \lambda_{J(u)} \mathrm{d} u\right\}
$$

is a $\left\{g_{t}\right\}$-martingale. Equivalently,

$$
\mathrm{E}\left(\exp \left\{\int_{0}^{t} \theta(u) \mathrm{d} S_{\mathcal{R}}(u)\right\} \mid J\right)=\exp \left\{\int_{0}^{t} \int_{0}^{\infty}\left(\exp \left\{\theta(u) R_{u}(x)\right\}-1\right) G_{J(u)}(\mathrm{d} x) \lambda_{J(u)} \mathrm{d} u\right\} .
$$

Proof. (i) For any $s<t$,

$$
\begin{aligned}
\mathrm{E}\left(S_{\mathcal{R}}(t) \mid g_{s}\right) & =S_{\mathcal{R}}(s)+\mathrm{E}\left(\sum_{l=N(s)+1}^{N(t)} R_{T_{l}}\left(U_{l}\right) \mid g_{s}\right) \\
& =S_{\mathcal{R}}(s)+\mathrm{E}\left(\sum_{l=1}^{N(t)-N(s)} R_{T_{N(s)+l}}\left(U_{N(s)+l}\right) \mid g_{s}\right)
\end{aligned}
$$

and

$$
\begin{aligned}
\mathrm{E}( & \left.\sum_{l=1}^{N(t)-N(s)} R_{T_{N(s)+l}}\left(U_{N(s)+l}\right) \mid g_{s}\right) \\
& =\sum_{m=1}^{\infty} \sum_{l=1}^{m} \mathrm{E}\left(\mathbf{1}_{\{N(t)-N(s)=m\}} R_{T_{N(s)+l}}\left(U_{N(s)+l}\right) \mid g_{s}\right) \\
& =\sum_{l=1}^{\infty} \mathrm{E}\left(\mathbf{1}_{\{N(t)-N(s) \geq l\}} R_{T_{N(s)+l}}\left(U_{N(s)+l}\right) \mid g_{s}\right) \\
& =\sum_{l=1}^{\infty} \mathrm{E}\left(\mathbf{1}_{\left\{\tilde{T}_{l} \leq t-s\right\}} \int_{0}^{\infty} R_{\tilde{T}_{l}+s}(x) G_{J\left(\tilde{T}_{l}+s\right)}(\mathrm{d} x) \mid g_{s}\right) \\
& =\mathrm{E}\left(\int_{0}^{\infty} \mathbf{1}_{(0, t-s]}(u) \int_{0}^{\infty} R_{s+u}(x) G_{J(s+u)}(\mathrm{d} x) \mathrm{d} \tilde{N}(u) \mid g_{s}\right),
\end{aligned}
$$


where

$$
\tilde{N}(u)=N(s+u)-N(s), \quad u \geq 0, \quad \text { and } \quad \tilde{T}_{l}=\inf \{u \geq 0, \tilde{N}(u)=l\} .
$$

Since $\tilde{N}(u)$ is a doubly stochastic Poisson process with intensity $\lambda_{J(s+u)}, \tilde{N}(u)-\lambda_{J(s+u)}$ is an $\left\{\tilde{\mathcal{F}}_{u}:=\widetilde{F}_{s+u}, u \geq 0\right\}$-martingale and so

$$
\begin{aligned}
& \mathrm{E}\left(\int_{0}^{\infty} \mathbf{1}_{(0, t-s]}(u) \int_{0}^{\infty} R_{s+u}(x) G_{J(s+u)}(\mathrm{d} x) \mathrm{d} \tilde{N}(u) \mid g_{s}\right) \\
& \quad=\mathrm{E}\left(\int_{0}^{\infty} \mathbf{1}_{(0, t-s]}(u) \int_{0}^{\infty} R_{s+u}(x) G_{J(s+u)}(\mathrm{d} x) \lambda_{J(s+u)} \mathrm{d} u \mid g_{s}\right) \\
& \quad=\int_{s}^{t} \int_{0}^{\infty} R_{u}(x) G_{J(u)}(\mathrm{d} x) \lambda_{J(u)} \mathrm{d} u .
\end{aligned}
$$

Therefore, $\left\{S_{\mathcal{R}}(t)-\int_{0}^{t} \int_{0}^{\infty} R_{u}(x) G_{J(u)}(\mathrm{d} x) \lambda_{J(u)} \mathrm{d} u\right\}$ is a $\left\{g_{t}\right\}$-martingale.

(ii) Set $L_{t}=\int_{0}^{t} \theta(u) \mathrm{d} S_{\mathcal{R}}(u)$. Applying Itô's formula (cf. [11, p. 242]) to $\exp \left\{L_{t}\right\}$, we have

$$
\begin{aligned}
\exp \left\{L_{t}\right\}= & 1+\int_{0}^{t} \exp \left\{L_{u-}\right\} \theta(u) \mathrm{d} S_{\mathcal{R}}(u)+\sum_{0<u \leq t} \exp \left\{L_{u-}\right\}\left(\exp \left\{\Delta L_{u}\right\}-1-\Delta L_{u}\right) \\
= & 1+\int_{0}^{t} \exp \left\{L_{u-}\right\} \theta(u) \mathrm{d} S_{\mathcal{R}}(u) \\
& +\sum_{0<u \leq t}^{t} \exp \left\{L_{u-}\right\}\left(\exp \left\{\theta(u) R_{u}\left(U_{N(u)}\right)\right\}-1-\theta(u) R_{u}\left(U_{N(u)}\right)\right) \mathbf{1}_{\{\Delta N(u)=1\}} \\
= & 1+\int_{0}^{t} \exp \left\{L_{u-}\right\} \theta(u) \mathrm{d} M_{u}+\int_{0}^{t} \int_{0}^{\infty} \exp \left\{L_{u-}\right\} \theta(u) R_{u}(x) G_{J(u)}(\mathrm{d} x) \lambda_{J(u)} \mathrm{d} u \\
& +\sum_{l=1}^{\infty} \exp \left\{L_{T_{l-1}}\right\}\left(\exp \left\{\theta\left(T_{l}\right) R_{T_{l}}\left(U_{l}\right)\right\}-1-\theta\left(T_{l}\right) R_{T_{l}}\left(U_{l}\right)\right) \mathbf{1}_{\left\{T_{l} \leq t\right\}},
\end{aligned}
$$

where $\Delta L_{u}=L_{u}-L_{u-}$. Therefore, the conditional expectation $\mathrm{E}\left(\exp \left\{L_{t}\right\} \mid J\right)$ of $\exp \left\{L_{t}\right\}$ with respect to $\sigma(J(s), s \geq 0)$ satisfies the following equation:

$$
\begin{aligned}
\mathrm{E}\left(\exp \left\{L_{t}\right\} \mid J\right)= & 1+\int_{0}^{t} \int_{0}^{\infty} \mathrm{E}\left(\exp \left\{L_{u-}\right\} \mid J\right) \theta(u) R_{u}(x) G_{J(u)}(\mathrm{d} x) \lambda_{J(u)} \mathrm{d} u \\
& +\sum_{l=1}^{\infty} \mathrm{E}\left(\exp \left\{L_{T_{l-1}}\right\}\left(\exp \left\{\theta\left(T_{l}\right) R_{T_{l}}\left(U_{l}\right)\right\}-1-\theta\left(T_{l}\right) R_{T_{l}}\left(U_{l}\right)\right) \mathbf{1}_{\left\{T_{l} \leq t\right\}} \mid J\right) .
\end{aligned}
$$


Since

$$
\begin{aligned}
& \sum_{l=1}^{\infty} \mathrm{E}\left(\exp \left\{L_{T_{l-1}}\right\}\left(\exp \left\{\theta\left(T_{l}\right) R_{T_{l}}\left(U_{l}\right)\right\}-1-\theta\left(T_{l}\right) R_{T_{l}}\left(U_{l}\right)\right) \mathbf{1}_{\left\{T_{l} \leq t\right\}} \mid J\right) \\
& \quad=\sum_{l=1}^{\infty} \int_{0}^{\infty} \mathrm{E}\left(\exp \left\{L_{T_{l-1}}\right\}\left(\exp \left\{\theta\left(T_{l}\right) R_{T_{l}}(x)\right\}-1-\theta\left(T_{l}\right) R_{T_{l}}(x)\right) \mathbf{1}_{\left\{T_{l} \leq t\right\}} G_{J\left(T_{l}\right)}(\mathrm{d} x) \mid J\right) \\
& =\mathrm{E}\left(\int_{0}^{t} \int_{0}^{\infty} \exp \left\{L_{u-}\right\}\left(\exp \left\{\theta(u) R_{u}(x)\right\}-1-\theta(u) R_{u}(x)\right) G_{J(u)}(\mathrm{d} x) \mathrm{d} N(u) \mid J\right) \\
& =\mathrm{E}\left(\int_{0}^{t} \int_{0}^{\infty} \exp \left\{L_{u-}\right\}\left(\exp \left\{\theta(u) R_{u}(x)\right\}-1-\theta(u) R_{u}(x)\right) G_{J(u)}(\mathrm{d} x) \lambda_{J(u)} \mathrm{d} u \mid J\right) \\
& =\int_{0}^{t} \int_{0}^{\infty} \mathrm{E}\left(\exp \left\{L_{u-}\right\} \mid J\right)\left(\exp \left\{\theta(u) R_{u}(x)\right\}-1-\theta(u) R_{u}(x)\right) G_{J(u)}(\mathrm{d} x) \lambda_{J(u)} \mathrm{d} u,
\end{aligned}
$$

we have

$$
\mathrm{E}\left(\exp \left\{L_{t}\right\} \mid J\right)=1+\int_{0}^{t} \int_{0}^{\infty} \mathrm{E}\left(\exp \left\{L_{u-}\right\} \mid J\right)\left(\exp \left\{\theta(u) R_{u}(x)\right\}-1\right) G_{J(u)}(\mathrm{d} x) \lambda_{J(u)} \mathrm{d} u
$$

which implies that

$$
\mathrm{E}\left(\exp \left\{L_{t}\right\} \mid J\right)=\exp \left\{\int_{0}^{t} \int_{0}^{\infty}\left(\exp \left\{\theta(u) R_{u}(x)\right\}-1\right) G_{J(u)}(\mathrm{d} x) \lambda_{J(u)} \mathrm{d} u\right\} .
$$

Corollary 2.1. If, for some $\delta>0$,

$$
\sup _{i \in E} \int_{0}^{\infty} \mathrm{e}^{\delta x} G_{i}(\mathrm{~d} x)<\infty
$$

then, for any $m \geq 1,0=t_{0}<t_{1}<\cdots<t_{m}$, and $\theta_{1}, \ldots, \theta_{m} \in(-\infty, \delta)$,

$$
\begin{aligned}
& \mathrm{E}\left(\exp \left\{\sum_{l=1}^{m} \theta_{l} \sum_{n=N\left(t_{l-1}\right)+1}^{N\left(t_{l}\right)} R_{T_{n}}\left(U_{n}\right)\right\} \mid J\right) \\
& \quad=\exp \left\{\sum_{l=1}^{m} \int_{t_{l-1}}^{t_{l}} \int_{0}^{\infty}\left(\exp \left\{\theta_{l} R_{u}(x)\right\}-1\right) G_{J(u)}(\mathrm{d} x) \lambda_{J(u)} \mathrm{d} u\right\} .
\end{aligned}
$$

Furthermore,

$$
\begin{aligned}
& \prod_{l=1}^{m} \inf _{i \in E} \mathrm{E}_{i}\left(\exp \left\{\int_{0}^{t_{l}-t_{l-1}} \int_{0}^{\infty}\left(\exp \left\{\theta_{l} R_{u+t_{l-1}}(x)\right\}-1\right) G_{J(u)}(\mathrm{d} x) \lambda_{J(u)} \mathrm{d} u\right\}\right) \\
& \leq \mathrm{E}\left(\exp \left\{\sum_{l=1}^{m} \theta_{l} \sum_{n=N\left(t_{l-1}\right)+1}^{N\left(t_{l}\right)} R_{T_{n}}\left(U_{n}\right)\right\}\right) \\
& \quad \leq \prod_{l=1}^{m} \sup _{i \in E} \mathrm{E}_{i}\left(\exp \left\{\int_{0}^{t_{l}-t_{l-1}} \int_{0}^{\infty}\left(\exp \left\{\theta_{l} R_{u+t_{l-1}}(x)\right\}-1\right) G_{J(u)}(\mathrm{d} x) \lambda_{J(u)} \mathrm{d} u\right\}\right),
\end{aligned}
$$

where $\mathrm{E}_{i}(\cdot):=\mathrm{E}(\cdot \mid J(0)=i)$. 
Proof. Take $\theta(u)=\sum_{l=1}^{m} \theta_{l} \mathbf{1}_{\left(t_{l-1}, t_{l}\right]}(u)$. Then Theorem 2.1(ii) yields (2.1). By the Markov property of $J$, it is easy to obtain (2.2).

Remark 2.1. Macci and Stabile [13] gave a representation of $\mathrm{E}\left(\exp \left\{\theta S_{\mathcal{R}}(t)\right\}\right)$. Our proof for the general result, (2.1), is based on Itô's formula.

\section{Large deviations}

In this section we establish a functional LDP for the process $S_{\mathcal{R}}(t)$. In order to obtain the LDP of $S_{\mathcal{R}}(t)$, Macci and Stabile [13] introduced the following two assumptions.

(H1) Let $\tilde{R}:[0, \infty) \rightarrow[0, \infty)$ be a measurable function. Then, for all $\varepsilon>0$, there exists $t_{\varepsilon}$ such that, for all $t \geq t_{\varepsilon}$, we have $\left|R_{t}(x)-\tilde{R}(x)\right| \leq \varepsilon(x+1)$ for all $x \geq 0$.

(H2) For all $r>0$,

$$
\sup _{i \in E} \int_{0}^{\infty} \mathrm{e}^{r x} G_{i}(\mathrm{~d} x)<\infty .
$$

We will prove that $\left\{S_{\mathcal{R}}(\alpha t) /\left.\alpha\right|_{t \in[0,1]}, \alpha>0\right\}$ satisfies a functional LDP in $D([0,1])$ under assumptions (H1) and (H2).

Let $Q=\left(q_{i j}\right)_{i, j \in E}$ be the intensity matrix of the Markov chain $\{J(t), t \geq 0\}$. For any vector $v=\left(v_{i}\right)_{i \in E}$, set $Q(v)=\left(q_{i j}+\delta_{i j} v_{i}\right)_{i, j \in E}$ and let $\Lambda(v)$ be the logarithm of the simple and positive eigenvalue of the exponential matrix $\mathrm{e}^{Q(v)}$. By applying the Feymnan-Kac formula we obtain (cf. [3] and [5, Corollary 4.2.27]), for any $j \in E$,

$$
\lim _{t \rightarrow \infty} \frac{1}{t} \log \mathrm{E}_{j}\left(\exp \left\{\int_{0}^{t} v_{J(u)} \mathrm{d} u\right\}\right)=\Lambda(v)
$$

Since, for any $j \in E$,

$$
\begin{aligned}
\inf _{i \in E} & \mathrm{E}_{i}\left(\exp \left\{\int_{0}^{t} \lambda_{J(u)} \int_{0}^{\infty}\left(\exp \left\{\theta R_{u}(x)\right\}-1\right) G_{J(u)}(\mathrm{d} x) \mathrm{d} u\right\}\right) \\
& \leq \mathrm{E}_{j}\left(\exp \left\{\theta S_{\mathcal{R}}(t)\right\}\right) \\
& \leq \sup _{i \in E} \mathrm{E}_{i}\left(\exp \left\{\int_{0}^{t} \lambda_{J(u)} \int_{0}^{\infty}\left(\exp \left\{\theta R_{u}(x)\right\}-1\right) G_{J(u)}(\mathrm{d} x) \mathrm{d} u\right\}\right),
\end{aligned}
$$

under assumptions (H1) and (H2), (3.1) implies that (see [13] for detail), for any $j \in E$ and any $\theta \in \mathbb{R}$,

$$
\lim _{t \rightarrow \infty} \frac{1}{t} \log \mathrm{E}_{j}\left(\exp \left\{\theta S_{\mathcal{R}}(t)\right\}\right)=\Lambda\left(\left(\lambda_{i} \int_{0}^{\infty}\left(\mathrm{e}^{\theta \tilde{R}(x)}-1\right) G_{i}(\mathrm{~d} x)\right)_{i \in E}\right) .
$$

Define

$$
\Lambda^{*}(x)=\sup _{\theta \in \mathbb{R}}\left\{\theta x-\Lambda\left(\left(\lambda_{i} \int_{0}^{\infty}\left(\mathrm{e}^{\theta \tilde{R}(z)}-1\right) G_{i}(\mathrm{~d} z)\right)_{i \in E}\right)\right\} .
$$

Lemma 3.1. Let assumptions (H1) and (H2) hold. Then, for any $m \geq 1$ and $0=t_{0} \leq t_{1}<$ $t_{2}<\cdots<t_{m} \leq 1,\left\{\left(S_{\mathcal{R}}\left(\alpha t_{1}\right) / \alpha, S_{\mathcal{R}}\left(\alpha t_{2}\right) / \alpha, \ldots, S_{\mathcal{R}}\left(\alpha t_{m}\right) / \alpha\right), \alpha>0\right\}$ satisfies the LDP speed $\alpha$ and rate function $I_{t_{1}, \ldots, t_{m}}^{(l d)}$ defined by

$$
I_{t_{1}, \ldots, t_{m}}^{(l d)}\left(x_{1}, \ldots, x_{m}\right)=\sum_{l=1}^{m}\left(t_{l}-t_{l-1}\right) \Lambda^{*}\left(\frac{x_{l}-x_{l-1}}{t_{l}-t_{l-1}}\right),
$$

where $x_{0}=0$. 
Proof. For any $\left(\theta_{1}, \theta_{2}, \ldots, \theta_{m}\right) \in \mathbb{R}^{m}$, by (2.2) we have

$$
\begin{aligned}
& \sum_{l=1}^{m}\left(t_{l}-t_{l-1}\right) \lim _{\alpha \rightarrow \infty} \frac{1}{\alpha\left(t_{l}-t_{l-1}\right)} \\
& \quad \times \log \inf _{i \in E} \mathrm{E}_{i}\left(\exp \left\{\int_{0}^{\alpha\left(t_{l}-t_{l-1}\right)} \lambda_{J(u)} \int_{0}^{\infty}\left(\exp \left\{\theta_{l} R_{u+\alpha t_{l-1}}(x)\right\}-1\right) G_{J(u)}(\mathrm{d} x) \mathrm{d} u\right\}\right) \\
& \leq \lim _{\alpha \rightarrow \infty} \frac{1}{\alpha} \log \mathrm{E}\left(\exp \left\{\sum_{l=1}^{m} \theta_{l} \sum_{n=N\left(\alpha t_{l-1}\right)+1}^{N\left(\alpha t_{l}\right)} R_{T_{n}}\left(U_{n}\right)\right\}\right) \\
& \leq \sum_{l=1}^{m}\left(t_{l}-t_{l-1}\right) \lim _{\alpha \rightarrow \infty} \frac{1}{\alpha\left(t_{l}-t_{l-1}\right)} \\
& \quad \times \log \sup _{i \in E} \mathrm{E}_{i}\left(\exp \left\{\int_{0}^{\alpha\left(t_{l}-t_{l-1}\right)} \lambda_{J(u)} \int_{0}^{\infty}\left(\exp \left\{\theta_{l} R_{u+\alpha t_{l-1}}(x)\right\}-1\right) G_{J(u)}(\mathrm{d} x) \mathrm{d} u\right\}\right) .
\end{aligned}
$$

Therefore, (3.2) implies that

$$
\begin{aligned}
\Lambda_{t_{1}, \ldots, t_{m}}\left(\theta_{1}, \ldots, \theta_{m}\right) & :=\lim _{\alpha \rightarrow \infty} \frac{1}{\alpha} \log \mathrm{E}\left(\exp \left\{\sum_{l=1}^{m} \theta_{l} \sum_{n=N\left(\alpha t_{l-1}\right)+1}^{N\left(\alpha t_{l}\right)} R_{T_{n}}\left(U_{n}\right)\right\}\right) \\
& =\sum_{l=1}^{m}\left(t_{l}-t_{l-1}\right) \Lambda\left(\left(\lambda_{i} \int_{0}^{\infty}\left(\exp \left\{\theta_{l} \tilde{R}(x)\right\}-1\right) G_{i}(\mathrm{~d} x)\right)_{i \in E}\right)
\end{aligned}
$$

By the Gärtner-Ellis theorem (cf. [4, p. 43]),

$$
\left(\frac{1}{\alpha} S_{\mathcal{R}}\left(\alpha t_{1}\right), \frac{1}{\alpha}\left(S_{\mathcal{R}}\left(\alpha t_{2}\right)-S_{\mathcal{R}}\left(\alpha t_{1}\right)\right), \ldots, \frac{1}{\alpha}\left(S_{\mathcal{R}}\left(\alpha t_{m}\right)-S_{\mathcal{R}}\left(\alpha t_{m-1}\right)\right)\right)
$$

satisfies the LDP with speed $\alpha$ and rate function $\Lambda_{t_{1}, \ldots, t_{m}}^{*}(\cdot)$ defined by

$$
\begin{aligned}
\Lambda_{t_{1}, \ldots, t_{m}}^{*}\left(x_{1}, \ldots, x_{m}\right) & =\sup _{\left(\theta_{1}, \ldots, \theta_{m}\right) \in \mathbb{R}^{m}}\left\{\sum_{l=1}^{m} \theta_{l} x_{l}-\Lambda_{t_{1}, \ldots, t_{m}}\left(\theta_{1}, \ldots, \theta_{m}\right)\right\} \\
& =\sum_{l=1}^{m}\left(t_{l}-t_{l-1}\right) \Lambda^{*}\left(\frac{x_{l}}{t_{l}-t_{l-1}}\right) .
\end{aligned}
$$

Now, since

$$
\begin{aligned}
& \left(\frac{1}{\alpha} S_{\mathcal{R}}\left(\alpha t_{1}\right), \frac{1}{\alpha} S_{\mathcal{R}}\left(\alpha t_{2}\right), \ldots, \frac{1}{\alpha} S_{\mathcal{R}}\left(\alpha t_{m}\right)\right) \\
& \quad=\left(\frac{1}{\alpha} S_{\mathcal{R}}\left(\alpha t_{1}\right), \frac{1}{\alpha}\left(S_{\mathcal{R}}\left(\alpha t_{2}\right)-S_{\mathcal{R}}\left(\alpha t_{1}\right)\right), \ldots, \frac{1}{\alpha}\left(S_{\mathcal{R}}\left(\alpha t_{m}\right)-S_{\mathcal{R}}\left(\alpha t_{m-1}\right)\right)\right) T,
\end{aligned}
$$

where the matrix $T=\left(t_{l k}\right)_{1 \leq l, k \leq m}$ satisfies $t_{l k}=1$ for $l \leq k$ and $t_{l k}=0$ for $l>k$, we obtain the conclusion of the lemma from the contract principle. 
Theorem 3.1. Let assumptions (H1) and (H2) hold. Then $\left\{\mathrm{P}\left(\left(S_{\mathcal{R}}(\alpha t) / \alpha\right)_{t \in[0,1]} \in \cdot\right), \alpha>0\right\}$ satisfies the LDP on $D([0,1])$ with speed $\alpha$ and rate function $I^{(l d)}$ defined by

$$
I^{(l d)}(f)= \begin{cases}\int_{0}^{1} \Lambda^{*}(\dot{f}(t)) \mathrm{d} t & \text { if } f(0)=0 \text { and } f \text { is absolutely continuous } \\ +\infty & \text { otherwise. }\end{cases}
$$

Proof. Firstly, using the standard argument (cf. [4, p. 180]), we obtain

$$
I^{(l d)}(f)=\sup _{m \geq 1} \sup _{0=t_{0} \leq t_{1}<t_{2}<\cdots<t_{m} \leq 1} \sum_{l=1}^{m}\left(t_{l}-t_{l-1}\right) \Lambda^{*}\left(\frac{f\left(t_{l}\right)-f\left(t_{l-1}\right)}{t_{l}-t_{l-1}}\right) .
$$

Therefore, by Lemma 1.2 and Lemma 3.1, we only need to prove the exponential tightness, i.e. for any $t \in[0,1]$ and any $\eta>0$,

$$
\lim _{\delta \downarrow 0} \lim _{\alpha \rightarrow \infty} \frac{1}{\alpha} \log \mathrm{P}\left(\frac{1}{\alpha} \sup _{t \leq s \leq t+\delta}\left|S_{\mathcal{R}}(\alpha t)-S_{\mathcal{R}}(\alpha s)\right| \geq \eta\right)=-\infty .
$$

By Theorem 2.1, for any $\beta \in \mathbb{R},\left(Z_{t}^{\beta}\right)^{-1} Z_{t+s}^{\beta}, s \geq 0$, is a martingale under probability $\mathrm{P}(\cdot \mid J)$, where

$$
Z_{t}^{\beta}:=\exp \left\{\beta S_{\mathcal{R}}(t)-\int_{0}^{t} \int_{0}^{\infty}\left(\exp \left\{\beta R_{u}(x)\right\}-1\right) G_{J(u)}(\mathrm{d} x) \lambda_{J(u)} \mathrm{d} u\right\} .
$$

Then, by the maximum inequality for a martingale, we have, for any $\beta>0$,

$$
\begin{aligned}
\frac{1}{\alpha} \log \mathrm{P} & \left(\frac{1}{\alpha} \sup _{t \leq s \leq t+\delta}\left|S_{\mathcal{R}}(\alpha t)-S_{\mathcal{R}}(\alpha s)\right| \geq \eta\right) \\
& =\frac{1}{\alpha} \log \mathrm{P}\left(\frac{1}{\alpha} \sup _{t \leq s \leq t+\delta}\left(S_{\mathcal{R}}(\alpha t)-S_{\mathcal{R}}(\alpha s)\right) \geq \eta\right) \\
& \leq \frac{1}{\alpha} \log \mathrm{E}\left(\mathrm{P}\left(\sup _{0 \leq s \leq \delta}\left(Z_{\alpha t}^{\beta}\right)^{-1} Z_{\alpha(t+s)}^{\beta} \geq \mathrm{e}^{\alpha \beta \eta-\alpha \delta C(\beta)} \mid J\right)\right) \\
& \leq \frac{1}{\alpha} \log \mathrm{E}\left(\mathrm{e}^{-\alpha \beta \eta+\alpha \delta C(\beta)} \mathrm{E}\left(\left(Z_{\alpha t}^{\beta}\right)^{-1} Z_{\alpha(t+\delta)}^{\beta} \mid J\right)\right) \\
& =-\beta \eta+\delta C(\beta),
\end{aligned}
$$

where

$$
C(\beta):=\sup _{i \in E} \lambda_{i} \int_{0}^{\infty}\left(\mathrm{e}^{\beta x}-1\right) G_{i}(\mathrm{~d} x) .
$$

Now letting $\alpha \rightarrow \infty$ firstly, $\delta \downarrow 0$ secondly, and $\beta \rightarrow \infty$ finally, we obtain (3.3).

\section{Moderate deviations}

In this section we establish a functional moderate deviation principle for the process $S_{\mathcal{R}}(t)$. Throughout this section, $\{a(t), t \geq 0\}$ denotes a positive function satisfying

$$
\lim _{t \rightarrow \infty} \frac{a(t)}{t}=0, \quad \lim _{t \rightarrow \infty} \frac{a(t)}{\sqrt{t}}=\infty .
$$


We introduce the following assumptions.

(H3) There exist two nonnegative measurable functions $\hat{R}(x)$ and $m(u)$ such that, for all $u \geq 0$ and $x>0,\left|R_{u}(x)-\hat{R}(x)\right| \leq m(u)(x+1)$ and

$$
\lim _{u \rightarrow \infty} m(u)=0, \quad \lim _{t \rightarrow \infty} \frac{1}{a(t)} \int_{0}^{t} m(u) \mathrm{d} u=0 .
$$

(H4) There exists $\delta>0$ such that

$$
\sup _{i \in E} \int_{0}^{\infty} \mathrm{e}^{\delta x} G_{i}(\mathrm{~d} x)<\infty .
$$

For example, if $R_{t}(x)=c\left(1-1 /(1+t)^{\gamma}\right) x^{\tau}$, where $c, \tau \in(0,1]$ and $\gamma>0$, then (H3) holds for $\hat{R}(x)=c x^{\tau}, m(u)=1 /(1+u)^{\gamma}$, and $a(t)=t^{\beta}$, where $\max \left\{1-\gamma, \frac{1}{2}\right\}<\beta<1$.

Since $\{J(t), t \geq 0\}$ is a uniformly ergodic Markov process with finite state space $E$, the following result is known (cf. [10] and [15]).

Lemma 4.1. Let $P(t)=\left(p_{i j}(t)\right)_{i, j \in E}=\mathrm{e}^{t Q}$ be the semigroup of the Markov chain $J$.

(i) There exists $c>0$ such that, for any function $f$ on $E$,

$$
\sup _{i \in E}\left|\sum_{k \in E} p_{i k}(t) f(k)-\sum_{j \in E} \pi_{j} f(j)\right| \leq \mathrm{e}^{-c t} \sup _{i \in E}|f(i)| .
$$

(ii) For any $j \in E$ and any function $f$ on $E$,

$$
\begin{aligned}
\lim _{t \rightarrow \infty} & \frac{1}{t} \mathrm{E}_{j}\left(\int_{0}^{t}\left(f(J(u))-\mathrm{E}_{\pi}(f(J(u)))\right) \mathrm{d} u\right)^{2} \\
& =2 \int_{0}^{\infty} \sum_{i \in E} \pi_{i}\left(f(i)-\sum_{k \in E} \pi_{k} f(k)\right) \sum_{k \in E} p_{i k}(u) f(k) \mathrm{d} u .
\end{aligned}
$$

(iii) For any $i \in E$ and any function $f$ on $E$,

$$
\begin{gathered}
\lim _{\alpha \rightarrow \infty} \frac{\alpha}{a^{2}(\alpha)} \log E_{i}\left(\exp \left\{\frac{a(\alpha)}{\alpha} \int_{0}^{\alpha t}\left(f(J(u))-\mathrm{E}_{\pi}(f(J(u)))\right) \mathrm{d} u\right\}\right) \\
=t \int_{0}^{\infty} \sum_{i \in E} \pi_{i}\left(f(i)-\sum_{k \in E} \pi_{k} f(k)\right) \sum_{k \in E} p_{i k}(u) f(k) \mathrm{d} u
\end{gathered}
$$

Remark 4.1. Set $\hat{R}_{i}=\int_{0}^{\infty} \hat{R}(x) G_{i}(\mathrm{~d} x)$. Then, by Lemma 4.1,

$$
\sigma_{1}^{2}:=\lim _{t \rightarrow \infty} \frac{1}{t} \mathrm{E}_{\pi}\left(\left(\int_{0}^{t}\left(\hat{R}_{J(u)} \lambda_{J(u)}-\mathrm{E}_{\pi}\left(\hat{R}_{J(u)} \lambda_{J(u)}\right)\right) \mathrm{d} u\right)^{2}\right)
$$

exists and

$$
\sigma_{1}^{2}=2 \int_{0}^{\infty} \sum_{j \in E} \pi_{j}\left(\lambda_{j} \hat{R}_{j}-\sum_{i \in E} \pi_{i} \lambda_{i} \hat{R}_{i}\right) \sum_{k \in E} p_{j k}(t) \lambda_{k} \hat{R}_{k} \mathrm{~d} t
$$


Lemma 4.2. Let assumptions (H3) and (H4) hold. Set $\bar{S}_{\mathcal{R}}(\cdot)=S_{\mathcal{R}}(\cdot)-\mathrm{E}_{\pi}\left(S_{\mathcal{R}}(\cdot)\right)$. Then, for any $j \in E, \theta \in \mathbb{R}$, and $t>0$,

$$
\lim _{\alpha \rightarrow \infty} \frac{\alpha}{a^{2}(\alpha)} \log \mathrm{E}_{j}\left(\exp \left\{\theta \frac{a(\alpha)}{\alpha} \bar{S}_{\mathcal{R}}(\alpha t)\right\}\right)=\frac{1}{2} \theta^{2} \sigma^{2} t,
$$

where $\sigma^{2}=\sigma_{1}^{2}+\sigma_{2}^{2}$ and

$$
\sigma_{2}^{2}=\sum_{i \in E}\left(\pi_{i} \lambda_{i} \int_{0}^{\infty} \hat{R}^{2}(x) G_{i}(\mathrm{~d} x)\right)
$$

Proof. By Theorem 2.1, for any $\theta \in \mathbb{R}, t>0$, and $\alpha>0$,

$$
\mathrm{E}_{\pi}\left(S_{\mathcal{R}}(\alpha t)\right)=\mathrm{E}_{\pi}\left(\int_{0}^{\alpha t} \lambda_{J(u)} \int_{0}^{\infty} R_{u}(x) G_{J(u)}(\mathrm{d} x) \mathrm{d} u\right)
$$

and, for all $j \in E$,

$$
\begin{aligned}
& \mathrm{E}_{j}\left(\exp \left\{\theta \frac{a(\alpha)}{\alpha} \bar{S}_{\mathcal{R}}(\alpha t)\right\}\right) \\
&=\mathrm{E}_{j}\left(\operatorname { e x p } \left\{\int _ { 0 } ^ { \alpha t } \left(\lambda_{J(u)} \int_{0}^{\infty}\left(\exp \left\{\theta \frac{a(\alpha)}{\alpha} R_{u}(x)\right\}-1\right) G_{J(u)}(\mathrm{d} x)\right.\right.\right. \\
&\left.\left.\left.\quad-\mathrm{E}_{\pi}\left(\theta \frac{a(\alpha)}{\alpha} \lambda_{J(u)} \int_{0}^{\infty} R_{u}(x) G_{J(u)}(\mathrm{d} x)\right)\right) \mathrm{d} u\right\}\right) .
\end{aligned}
$$

Therefore,

$$
\inf _{i \in E} \mathrm{E}_{i}(\xi \eta \zeta) \leq \mathrm{E}_{j}\left(\exp \left\{\theta \frac{a(\alpha)}{\alpha} \bar{S}_{\mathcal{R}}(\alpha t)\right\}\right) \leq \sup _{i \in E} \mathrm{E}_{i}(\xi \eta \zeta)
$$

where

$$
\begin{gathered}
\xi=\exp \left\{\int _ { 0 } ^ { \alpha t } \theta \frac { a ( \alpha ) } { \alpha } \left(\lambda_{J(u)} \int_{0}^{\infty} R_{u}(x) G_{J(u)}(\mathrm{d} x)\right.\right. \\
\left.\left.-\mathrm{E}_{\pi}\left(\lambda_{J(u)} \int_{0}^{\infty} R_{u}(x) G_{J(u)}(\mathrm{d} x)\right)\right) \mathrm{d} u\right\}, \\
\eta=\exp \left\{\int_{0}^{\alpha t} \lambda_{J(u)} \int_{0}^{\infty} \frac{1}{2}\left(\theta \frac{a(\alpha)}{\alpha} R_{u}(x)\right)^{2} G_{J(u)}(\mathrm{d} x) \mathrm{d} u\right\}, \\
\zeta=\exp \left\{\int_{0}^{\alpha t} \lambda_{J(u)} \int_{0}^{\infty} \sum_{l=3}^{\infty}\left(\frac{1}{l !}\left(\theta \frac{a(\alpha)}{\alpha} R_{u}(x)\right)^{l}\right) G_{J(u)}(\mathrm{d} x) \mathrm{d} u\right\} .
\end{gathered}
$$

By Hölder's inequality,

$$
\sup _{i \in E} \mathrm{E}_{i}(\xi \eta \zeta) \leq\left(\sup _{i \in E} \mathrm{E}_{i}\left(\xi^{p}\right)\right)^{1 / p}\left(\sup _{i \in E} \mathrm{E}_{i}\left(\eta^{q}\right)\right)^{1 / q}\left(\sup _{i \in E} \mathrm{E}_{i}\left(\zeta^{r}\right)\right)^{1 / r},
$$

where $1 / p+1 / q+1 / r=1, p>1, q>1$, and $r>1$. From assumption (H3), we have $R_{u}(x) \leq \hat{R}(x)+m(u)(x+1),-R_{u}(x) \leq-\hat{R}(x)+m(u)(x+1)$, and $0 \leq R_{u}(x), \hat{R}(x) \leq x$. 
Therefore,

$$
\begin{aligned}
& \frac{\alpha}{a^{2}(\alpha)} \log \left(\sup _{i \in E} \mathrm{E}_{i}\left(\xi^{p}\right)\right)^{1 / p} \\
& \leq \frac{\alpha}{p a^{2}(\alpha)} \log \sup _{i \in E} \mathrm{E}_{i}\left(\exp \left\{\frac{p \theta a(\alpha)}{\alpha} \int_{0}^{\alpha t}\left(\lambda_{J(u)} \hat{R}_{J(u)}-\mathrm{E}_{\pi}\left(\lambda_{J(u)} \hat{R}_{J(u)}\right)\right) \mathrm{d} u\right\}\right) \\
& \quad+\frac{2|\theta| A}{a(\alpha)} \int_{0}^{\alpha t} m(u) \mathrm{d} u
\end{aligned}
$$

and

$$
\begin{aligned}
\frac{\alpha}{a^{2}(\alpha)} & \log \left(\sup _{i \in E} \mathrm{E}_{i}\left(\eta^{q}\right)\right)^{1 / q} \\
\leq & \frac{\alpha}{q a^{2}(\alpha)} \log \sup _{i \in E} \mathrm{E}_{i}\left(\exp \left\{\frac{q}{2}\left(\frac{\theta a(\alpha)}{\alpha}\right)^{2} \int_{0}^{\alpha t} \lambda_{J(u)} \int_{0}^{\infty} \hat{R}^{2}(x) G_{J(u)}(\mathrm{d} x) \mathrm{d} u\right\}\right) \\
& +\frac{\theta^{2} A}{\alpha} \int_{0}^{\alpha t} m(u) \mathrm{d} u+\frac{\theta^{2} A}{2 \alpha} \int_{0}^{\alpha t} m^{2}(u) \mathrm{d} u \\
= & \frac{\alpha}{q a^{2}(\alpha)} \log \sup _{i \in E} \mathrm{E}_{i}\left(\exp \left\{\frac{q}{2}\left(\frac{\theta a(\alpha)}{\alpha}\right)^{2} \int_{0}^{\alpha t}\left(\lambda_{J(u)} \int_{0}^{\infty} \hat{R}^{2}(x) G_{J(u)}(\mathrm{d} x)-\sigma_{2}^{2}\right) \mathrm{d} u\right\}\right) \\
& +\frac{\theta^{2} A}{\alpha} \int_{0}^{\alpha t} m(u) \mathrm{d} u+\frac{\theta^{2} A}{2 \alpha} \int_{0}^{\alpha t} m^{2}(u) \mathrm{d} u+\frac{1}{2} \theta^{2} \sigma_{2}^{2} t,
\end{aligned}
$$

where $A=\sup _{i \in E}\left\{\lambda_{i} \int_{0}^{\infty}(x+1)^{2} G_{i}(\mathrm{~d} x)\right\}$. Then, applying Lemma 4.1, we have

$$
\limsup _{\alpha \rightarrow \infty} \frac{\alpha}{a^{2}(\alpha)} \log \left(\sup _{i \in E} \mathrm{E}_{i}\left(\xi^{p}\right)\right)^{1 / p} \leq \frac{1}{2} p \theta^{2} \sigma_{1}^{2} t
$$

and

$$
\limsup _{\alpha \rightarrow \infty} \frac{\alpha}{a^{2}(\alpha)} \log \left(\sup _{i \in E} \mathrm{E}_{i}\left(\eta^{q}\right)\right)^{1 / q} \leq \frac{1}{2} \theta^{2} \sigma_{2}^{2} t .
$$

Since, for any $r, \theta \in \mathbb{R}$, there exists a constant $M>0$ such that, for all $\alpha \geq M$,

$$
\begin{aligned}
0<\zeta^{r} & \leq \exp \left\{|r| \int_{0}^{\alpha t} \lambda_{J(u)} \int_{0}^{\infty} \sum_{l=3}^{\infty} \frac{1}{l !}\left(\frac{|\theta| x a(\alpha)}{\alpha}\right)^{l} G_{J(u)}(\mathrm{d} x) \mathrm{d} u\right\} \\
& \leq \exp \left\{6|r| \alpha t\left(\frac{2|\theta| a(\alpha)}{\delta \alpha}\right)^{3} \sup _{i \in E} \lambda_{i} \int_{0}^{\infty} \mathrm{e}^{\delta x} G_{i}(\mathrm{~d} x)\right\},
\end{aligned}
$$

where $\delta>0$ satisfies assumption (H4), then, for any $r, \theta \in \mathbb{R}$,

$$
\limsup _{\alpha \rightarrow \infty} \frac{\alpha}{a^{2}(\alpha)} \log \left(\sup _{i \in E} \mathrm{E}_{i}\left(\zeta^{r}\right)\right)^{1 / r}=0 .
$$

Therefore, for all $p>1$,

$$
\limsup _{\alpha \rightarrow \infty} \frac{\alpha}{a^{2}(\alpha)} \log \left(\sup _{i \in E} \mathrm{E}_{i}(\xi \eta \zeta)\right) \leq \frac{1}{2} p \theta^{2} \sigma_{1}^{2} t+\frac{1}{2} \theta^{2} \sigma_{2}^{2} t,
$$


which implies the following upper bound:

$$
\limsup _{\alpha \rightarrow \infty} \frac{\alpha}{a^{2}(\alpha)} \log \sup _{i \in E} \mathrm{E}_{i}\left(\exp \left\{\theta \frac{a(\alpha)}{\alpha} \bar{S}_{\mathcal{R}}(\alpha t)\right\}\right) \leq \frac{1}{2} \theta^{2} \sigma_{1}^{2} t+\frac{1}{2} \theta^{2} \sigma_{2}^{2} t=\frac{1}{2} \theta^{2} \sigma^{2} t .
$$

Now let us show the lower bound. Again, by Hölder's inequality,

$$
\inf _{i \in E} \mathrm{E}_{i}(\xi) \leq\left(\inf _{i \in E} \mathrm{E}_{i}(\xi \eta \zeta)^{q}\right)^{1 / q}\left(\sup _{i \in E} \mathrm{E}_{i}\left(\eta^{-p}\right)\right)^{1 / p}\left(\sup _{i \in E} \mathrm{E}_{i}\left(\zeta^{-r}\right)\right)^{1 / r},
$$

where $1 / p+1 / q+1 / r=1, p>1, q>1$, and $r>1$. From assumption (H3), we also have $R_{u}(x)+m(u)(x+1) \geq \hat{R}(x)$, and so, for all $u \geq 0, R_{u}^{2}(x) \geq \hat{R}^{2}(x)-\left(2 m(u)+m(u)^{2}\right)(x+1)^{2}$. Therefore,

$$
\begin{aligned}
& \frac{\alpha}{a^{2}(\alpha)} \log \left(\sup _{i \in E} \mathrm{E}_{i}\left(\eta^{-p}\right)\right)^{1 / p} \\
& \leq \frac{\alpha}{p a^{2}(\alpha)} \log \sup _{i \in E} \mathrm{E}_{i}\left(\exp \left\{-\frac{p}{2}\left(\theta \frac{a(\alpha)}{\alpha}\right)^{2} \int_{0}^{\alpha t}\left(\lambda_{J(u)} \int_{0}^{\infty} \hat{R}^{2}(x) G_{J(u)}(\mathrm{d} x)-\sigma_{2}^{2}\right) \mathrm{d} u\right\}\right) \\
& \quad+\frac{\theta^{2} A}{\alpha} \int_{0}^{\alpha t} m(u) \mathrm{d} u+\frac{\theta^{2} A}{2 \alpha} \int_{0}^{\alpha t} m^{2}(u) \mathrm{d} u-\frac{1}{2} \theta^{2} \sigma_{2}^{2} t
\end{aligned}
$$

and

$$
\begin{aligned}
\frac{\alpha}{a^{2}(\alpha)} & \log \left(\inf _{i \in E} \mathrm{E}_{i}(\xi)\right) \\
\geq & \frac{\alpha}{a^{2}(\alpha)} \log \inf _{i \in E} \mathrm{E}_{i}\left(\exp \left\{\int_{0}^{\alpha t} \theta \frac{a(\alpha)}{\alpha}\left(\lambda_{J(u)} \hat{R}_{J(u)}-\mathrm{E}_{\pi}\left(\lambda_{J(u)} \hat{R}_{J(u)}\right)\right) \mathrm{d} u\right\}\right) \\
& \quad-2 \theta \sup _{i \in E}\left(\lambda_{i} \hat{R}_{i}\right) \frac{1}{a(\alpha)} \int_{0}^{\alpha t} m(u) \mathrm{d} u .
\end{aligned}
$$

Then by Lemma 4.1 we have

$$
\limsup _{\alpha \rightarrow \infty} \frac{\alpha}{a^{2}(\alpha)} \log \left(\sup _{i \in E} \mathrm{E}_{i}\left(\eta^{-p}\right)\right)^{1 / p} \leq-\frac{1}{2} \theta^{2} \sigma_{2}^{2} t
$$

and

$$
\liminf _{\alpha \rightarrow \infty} \frac{\alpha}{a^{2}(\alpha)} \log \left(\inf _{i \in E} \mathrm{E}_{i}(\xi)\right) \geq \frac{1}{2} \theta^{2} \sigma_{1}^{2} t .
$$

Therefore, for any $q>1$,

$$
\liminf _{\alpha \rightarrow \infty} \frac{\alpha}{a^{2}(\alpha)} \log \left(\inf _{i \in E} \mathrm{E}_{i}(\xi \eta \zeta)^{q}\right)^{1 / q} \geq \frac{1}{2} \theta^{2}\left(\sigma_{1}^{2}+\sigma_{2}^{2}\right) t=\frac{1}{2} \theta^{2} \sigma^{2} t,
$$

that is, for any $q>1$,

$$
\liminf _{\alpha \rightarrow \infty} \frac{\alpha}{q a^{2}(\alpha)} \log \inf _{i \in E} \mathrm{E}_{i}\left(\exp \left\{\theta \frac{q a(\alpha)}{\alpha} \bar{S}_{\mathcal{R}}(\alpha t)\right\}\right) \geq \frac{1}{2} q \theta^{2}\left(\sigma_{1}^{2}+\sigma_{2}^{2}\right) t=\frac{q}{2} \theta^{2} \sigma^{2} t,
$$

which implies the following lower bound:

$$
\liminf _{\alpha \rightarrow \infty} \frac{\alpha}{a^{2}(\alpha)} \log \inf _{i \in E} E_{i}\left(\exp \left\{\theta \frac{a(\alpha)}{\alpha} \bar{S}_{\mathcal{R}}(\alpha t)\right\}\right) \geq \frac{1}{2} \theta^{2} \sigma^{2} t
$$


In conclusion, for all $j \in E, \theta \in \mathbb{R}$, and $t>0$, we have

$$
\lim _{\alpha \rightarrow \infty} \frac{\alpha}{a^{2}(\alpha)} \log \mathrm{E}_{j}\left(\exp \left\{\theta \frac{a(\alpha)}{\alpha} \bar{S}_{\mathcal{R}}(\alpha t)\right\}\right)=\frac{1}{2} \theta^{2} \sigma^{2} t
$$

Lemma 4.3. Let assumptions (H3) and (H4) hold. Then, for any $\alpha>0$ and $0=t_{0} \leq t_{1}<$ $t_{2}<\cdots<t_{m} \leq 1,\left(\bar{S}_{\mathcal{R}}\left(\alpha t_{1}\right) / a(\alpha), \bar{S}_{\mathcal{R}}\left(\alpha t_{2}\right) / a(\alpha), \ldots, \bar{S}_{\mathcal{R}}\left(\alpha t_{m}\right) / a(\alpha)\right)$ satisfies the LDP with speed $a^{2}(\alpha) / \alpha$ and rate function $I_{t_{1}, \ldots, t_{m}}^{(m d)}$ defined by

$$
I_{t_{1}, \ldots, t_{m}}^{(m d)}\left(x_{1}, \ldots, x_{m}\right)=\frac{1}{2 \sigma^{2}} \sum_{l=1}^{m} \frac{\left(x_{l}-x_{l-1}\right)^{2}}{t_{l}-t_{l-1}}
$$

where $x_{0}=0$.

Proof. According to (2.2), for any $\left(\theta_{1}, \theta_{2}, \ldots, \theta_{m}\right) \in \mathbb{R}^{m}$ and any $\alpha>0$ satisfying $\max _{1 \leq l \leq m}\left|\theta_{l}\right| a(\alpha) / \alpha<\delta$,

$$
\begin{aligned}
& \prod_{l=1}^{m} \inf _{i \in E} \mathrm{E}_{i}\left(\operatorname { e x p } \left\{\int _ { 0 } ^ { \alpha ( t _ { l } - t _ { l - 1 } ) } \left(\lambda_{J(u)} \int_{0}^{\infty}\left(\exp \left\{\theta_{l} \frac{a(\alpha)}{\alpha} R_{u+\alpha t_{l-1}}(x)\right\}-1\right) G_{J(u)}(\mathrm{d} x)\right.\right.\right. \\
& \left.\left.\left.\quad-\mathrm{E}_{\pi}\left(\theta_{l} \frac{a(\alpha)}{\alpha} \lambda_{J(u)} \int_{0}^{\infty} R_{u+\alpha t_{l-1}}(x) G_{J(u)}(\mathrm{d} x)\right)\right) \mathrm{d} u\right\}\right) \\
& \leq \mathrm{E}_{j}\left(\exp \left\{\sum_{l=1}^{m} \theta_{l} \frac{a(\alpha)}{\alpha}\left(\bar{S}_{\mathcal{R}}\left(\alpha t_{l}\right)-\bar{S}_{\mathcal{R}}\left(\alpha t_{l-1}\right)\right)\right\}\right) \\
& \leq \prod_{l=1}^{m} \sup _{i \in E} \mathrm{E}_{i}\left(\operatorname { e x p } \left\{\int _ { 0 } ^ { \alpha ( t _ { l } - t _ { l - 1 } ) } \left(\lambda_{J(u)} \int_{0}^{\infty}\left(\exp \left\{\theta_{l} \frac{a(\alpha)}{\alpha} R_{u+\alpha t_{l-1}}(x)\right\}-1\right) G_{J(u)}(\mathrm{d} x)\right.\right.\right. \\
& \left.\left.\left.\quad-\mathrm{E}_{\pi}\left(\theta_{l} \frac{a(\alpha)}{\alpha} \lambda_{J(u)} \int_{0}^{\infty} R_{u+\alpha t_{l-1}}(x) G_{J(u)}(\mathrm{d} x)\right)\right) \mathrm{d} u\right\}\right) .
\end{aligned}
$$

Therefore, by Lemma 4.2 we have

$$
\lim _{\alpha \rightarrow \infty} \frac{a(\alpha)}{\alpha} \log \mathrm{E}_{j}\left(\exp \left\{\sum_{l=1}^{m} \theta_{l} \frac{a(\alpha)}{\alpha}\left(\bar{S}_{\mathcal{R}}\left(\alpha t_{l}\right)-\bar{S}_{\mathcal{R}}\left(\alpha t_{l-1}\right)\right)\right\}\right)=\frac{\sigma^{2}}{2} \sum_{l=1}^{m}\left(t_{l}-t_{l-1}\right) \theta_{l}^{2},
$$

and so, by the Gärtner-Ellis theorem,

$$
\left(\frac{\bar{S}_{\mathcal{R}}\left(\alpha t_{1}\right)}{a(\alpha)}, \bar{S}_{\mathcal{R}}\left(\alpha t_{2}\right)-\frac{\bar{S}_{\mathcal{R}}\left(\alpha t_{1}\right)}{a(\alpha)}, \ldots, \frac{\bar{S}_{\mathcal{R}}\left(\alpha t_{m}\right)-\bar{S}_{\mathcal{R}}\left(\alpha t_{m-1}\right)}{a(\alpha)}\right)
$$

satisfies the LDP with speed $a^{2}(\alpha) / \alpha$ and rate function $J_{t_{1}, \ldots, t_{m}}^{(m d)}$ defined by

$$
J_{t_{1}, \ldots, t_{m}}^{(m d)}\left(x_{1}, \ldots, x_{m}\right)=\frac{1}{2 \sigma^{2}} \sum_{l=1}^{m} \frac{x_{l}^{2}}{\left(t_{l}-t_{l-1}\right)},
$$

which implies the conclusion of the lemma from the contract principle. 
Theorem 4.1. Let assumptions (H3) and (H4) hold. Then

$$
\left\{\mathrm{P}\left(\left.\frac{\bar{S}_{\mathcal{R}}(\alpha t)}{a(\alpha)}\right|_{t \in[0,1]} \in \cdot\right), \alpha>0\right\}
$$

and

$$
\left\{\mathrm{P}\left(\left.\frac{S_{\mathcal{R}}(\alpha t)-\alpha t \sum_{i \in E} \pi_{i} \lambda_{i} \hat{R}_{i}}{a(\alpha)}\right|_{t \in[0,1]} \in \cdot\right), \alpha>0\right\}
$$

satisfy the LDP on $D([0,1])$ with speed $a^{2}(\alpha) / \alpha$ and rate function $I^{(m d)}$ defined by

$$
I^{(m d)}(f)= \begin{cases}\frac{1}{2 \sigma^{2}} \int_{0}^{1}(\dot{f}(t))^{2} \mathrm{~d} t & \text { if } f(0)=0 \text { and } f \text { is absolutely continuous } \\ +\infty & \text { otherwise. }\end{cases}
$$

Proof. Firstly, it follows from assumption (H3) that

$$
\lim _{\alpha \rightarrow \infty} \frac{\sup _{t \in[0,1]}\left|\mathrm{E}\left(S_{\mathcal{R}}(\alpha t)\right)-\alpha t \sum_{i \in E} \pi_{i} \lambda_{i} \hat{R}_{i}\right|}{a(\alpha)}=0
$$

therefore, we prove only the first statement. Using the standard argument (cf. [4, p. 180]), it is easy to obtain

$$
I^{(m d)}(f)=\sup _{m \geq 1} \sup _{0=t_{0} \leq t_{1}<t_{2}<\cdots<t_{m} \leq 1} \frac{1}{2 \sigma^{2}} \sum_{l=1}^{m} \frac{\left(f\left(t_{l}\right)-f\left(t_{l-1}\right)\right)^{2}}{t_{l}-t_{l-1}} .
$$

Therefore, by Lemma 1.2 and Lemma 4.3, we only need to prove the exponential tightness, i.e. for any $t \in[0,1]$ and any $\eta>0$,

$$
\lim _{\delta \downarrow 0} \lim _{\alpha \rightarrow \infty} \frac{\alpha}{a^{2}(\alpha)} \log \mathrm{P}\left(\frac{1}{a(\alpha)} \sup _{t \leq s \leq t+\delta}\left|\bar{S}_{\mathcal{R}}(\alpha t)-\bar{S}_{\mathcal{R}}(\alpha s)\right| \geq \eta\right)=-\infty .
$$

By Theorem 2.1, for any $\beta \in \mathbb{R},\left(Z_{t}^{\beta}\right)^{-1} Z_{t+s}^{\beta}, s \geq 0$, is a martingale under probability $\mathrm{P}(\cdot \mid J)$. Then by the maximum inequality for a martingale we have, for any $\beta>0$,

$$
\begin{aligned}
\frac{\alpha}{a^{2}(\alpha)} & \log \mathrm{P}\left(\frac{1}{a(\alpha)} \sup _{t \leq s \leq t+\delta}\left(\bar{S}_{\mathcal{R}}(\alpha t)-\bar{S}_{\mathcal{R}}(\alpha s)\right) \geq \eta\right) \\
& \leq \frac{\alpha}{a^{2}(\alpha)} \log \mathrm{E}\left(\mathrm{P}\left(\sup _{0 \leq s \leq \delta}\left(Z_{\alpha t}^{a(\alpha) \beta / \alpha}\right)^{-1} Z_{\alpha(t+s)}^{a(\alpha) \beta / \alpha} \geq \exp \left\{\frac{a^{2}(\alpha) \beta \eta}{\alpha}-\alpha \delta C(\alpha, \beta)\right\} \mid J\right)\right) \\
& \leq \frac{\alpha}{a^{2}(\alpha)} \log \mathrm{E}\left(\exp \left\{-\frac{a^{2}(\alpha) \beta \eta}{\alpha}+\alpha \delta C(\alpha, \beta)\right\} \mathrm{E}\left(\left(Z_{\alpha t}^{\beta}\right)^{-1} Z_{\alpha(t+\delta)}^{\beta} \mid J\right)\right) \\
& =-\beta \eta+\frac{\alpha^{2}}{a^{2}(\alpha)} \delta C(\alpha, \beta),
\end{aligned}
$$

where

$$
C(\alpha, \beta):=\sup _{i \in E} \lambda_{i} \int_{0}^{\infty}\left(\mathrm{e}^{a(\alpha) \beta x / \alpha}-1-\frac{a(\alpha) \beta x}{\alpha}\right) G_{i}(\mathrm{~d} x)=O\left(\frac{a^{2}(\alpha)}{\alpha^{2}}\right) .
$$


Now letting $\alpha \rightarrow \infty$ firstly, $\delta \downarrow 0$ secondly, and $\beta \rightarrow \infty$ finally, we obtain

$$
\lim _{\delta \downarrow 0} \lim _{\alpha \rightarrow \infty} \frac{\alpha}{a^{2}(\alpha)} \log \mathrm{P}\left(\frac{1}{a(\alpha)} \sup _{t \leq s \leq t+\delta}\left(\bar{S}_{\mathcal{R}}(\alpha t)-\bar{S}_{\mathcal{R}}(\alpha s)\right) \geq \eta\right)=-\infty .
$$

Similarly, we can obtain

$$
\lim _{\delta \downarrow 0} \lim _{\alpha \rightarrow \infty} \frac{\alpha}{a^{2}(\alpha)} \log \mathrm{P}\left(\frac{1}{a(\alpha)} \inf _{t \leq s \leq t+\delta}\left(\bar{S}_{\mathcal{R}}(\alpha t)-\bar{S}_{\mathcal{R}}(\alpha s)\right) \leq-\eta\right)=-\infty .
$$

The proof of (4.1) is completed.

By $\inf _{\sup _{0 \leq t \leq 1}|f(t)| \geq r} I^{(m d)}(f)=r^{2} / 2 \sigma^{2}$ for any $r>0$, we have the following corollary, which gives a moderate deviation estimate of the aggregate claims process.

Corollary 4.1. Let assumptions (H3) and (H4) hold. Then, for any $r>0$,

$$
\lim _{\alpha \rightarrow \infty} \frac{\alpha}{a^{2}(\alpha)} \log \mathrm{P}\left(\sup _{t \in[0,1]}\left|S_{\mathcal{R}}(\alpha t)-\alpha t \sum_{i \in E} \pi_{i} \lambda_{i} \hat{R}_{i}\right| \geq r a(\alpha)\right)=-\frac{r^{2}}{2 \sigma^{2}} .
$$

\section{An estimate for the ruin probability}

The ruin time and the ruin probability are defined by

$$
\tau_{x}=\inf \left\{t \geq 0 ; X_{\mathcal{R}}^{x}(t)<0\right\} \quad \text { and } \quad \psi(x)=\mathrm{P}\left(\tau_{x}<\infty\right) .
$$

Macci and Stabile [13] proved, by the large deviation approach (cf. [8]), that if

$$
(1+\eta) \sum_{i \in E} \pi_{i} \lambda_{i} \int_{0}^{\infty} \tilde{R}(x) G_{i}(\mathrm{~d} x)-(\eta-\kappa) \sum_{i \in E} \pi_{i} \lambda_{i} \mu_{i}>\sum_{i \in E} \pi_{i} \lambda_{i} \int_{0}^{\infty} \tilde{R}(x) G_{i}(\mathrm{~d} x)
$$

holds then there exists $w_{\mathcal{R}}>0$ such that

$$
\lim _{x \rightarrow \infty} \frac{1}{x} \log \psi(x)=-w_{\mathcal{R}}
$$

In this section we give an estimate for the ruin probability (Lundberg's estimate) using the exponential martingale method.

Theorem 5.1. Let assumptions (H1) and (H2) hold. Set

$$
R:=\sup \left\{r>0 ; \inf _{t \geq 0}\left(r p_{\mathcal{R}}(t)-t \sup _{i \in E} \lambda_{i} \int_{0}^{\infty}\left(\mathrm{e}^{r x}-1\right) G_{i}(\mathrm{~d} x)\right) \geq 0\right\} .
$$

Then

$$
\psi(x) \leq \mathrm{e}^{-R x}
$$

Proof. Without loss of generality, we assume that $0<R<\infty$. By Theorem 2.1, for any $\beta \in \mathbb{R}$

$$
Z_{t}^{\beta}:=\exp \left\{\beta S_{\mathcal{R}}(t)-\int_{0}^{t} \int_{0}^{\infty}\left(\mathrm{e}^{\beta R_{u}(x)}-1\right) G_{J(u)}(\mathrm{d} x) \lambda_{J(u)} \mathrm{d} u\right\}
$$


is a martingale under probability $\mathrm{P}(\cdot \mid J)$. Therefore, by Doob's stopping time theorem, we have, for any $\beta>0$ and any $t \in[0, \infty), \mathrm{E}\left(Z_{\tau_{x} \wedge t}^{\beta}\right)=1$, which implies that $\mathrm{E}\left(Z_{\tau_{x}}^{\beta} \mathbf{1}_{\left\{\tau_{x}<\infty\right\}}\right)=1$. Therefore,

$$
\begin{aligned}
\psi(x) & =\mathrm{P}\left(S_{\mathcal{R}}\left(\tau_{x}\right) \geq x+p_{\mathcal{R}}\left(\tau_{x}\right), \tau_{x}<\infty\right) \\
\leq & \mathrm{e}^{-R x} \mathrm{E}\left(Z _ { \tau _ { x } } ^ { R } \operatorname { e x p } \left\{-R p_{\mathcal{R}}\left(\tau_{x}\right)+\int_{0}^{\tau_{x}} \int_{0}^{\infty}\left(\exp \left\{R R_{u}(x)\right\}-1\right)\right.\right. \\
& \left.\left.\times G_{J(u)}(\mathrm{d} x) \lambda_{J(u)} \mathrm{d} u\right\} \mathbf{1}_{\left\{\tau_{x}<\infty\right\}}\right) \\
\leq & \mathrm{e}^{-R x} \mathrm{E}\left(Z_{\tau_{x}}^{R} \exp \left\{-\left(R p_{\mathcal{R}}\left(\tau_{x}\right)-\tau_{x} \sup _{i \in E} \lambda_{i} \int_{0}^{\infty}\left(\mathrm{e}^{R x}-1\right) G_{i}(\mathrm{~d} x)\right)\right\} \mathbf{1}_{\left\{\tau_{x}<\infty\right\}}\right) \\
\leq & \mathrm{e}^{-R x} \mathrm{E}\left(Z_{\tau_{x}}^{R} \mathbf{1}_{\left\{\tau_{x}<\infty\right\}}\right) \\
& =\mathrm{e}^{-R x} .
\end{aligned}
$$

Remark 5.1. Assume that $\varrho x \leq R_{S}(x) \leq x$ for some constant $0 \leq \varrho \leq 1$. Then

$$
\begin{aligned}
& r p_{\mathcal{R}}(t)-t \sup _{i \in E} \lambda_{i} \int_{0}^{\infty}\left(\mathrm{e}^{r x}-1\right) G_{i}(\mathrm{~d} x) \\
& \quad \geq r t\left((\kappa-\eta+(1+\eta) \varrho) \sum_{i \in E} \pi_{i} \lambda_{i} \mu_{i}-\sup _{i \in E} \lambda_{i} \int_{0}^{\infty} r^{-1}\left(\mathrm{e}^{r x}-1\right) G_{i}(\mathrm{~d} x)\right) .
\end{aligned}
$$

Since $\sup _{i \in E} \lambda_{i} \int_{0}^{\infty} r^{-1}\left(\mathrm{e}^{r x}-1\right) G_{i}(\mathrm{~d} x) \longrightarrow \sup _{i \in E} \lambda_{i} \mu_{i}$ as $r \rightarrow 0$, we have $R>0$ if $(\kappa-$ $\eta+(1+\eta) \varrho) \sum_{i \in E} \pi_{i} \lambda_{i} \mu_{i}>\sup _{i \in E} \lambda_{i} \mu_{i}$.

Here we present a numerical example in which we calculate $R$ in Theorem 5.1. We consider the proportional policy (see [9, p. 509] and [13]), i.e. $R_{t}(x)=b_{t} x$ for some $b_{t} \in[0,1]$, and assume that $\lim _{t \rightarrow \infty} b_{t}=b_{\infty} \in[0,1]$.

Example 5.1. Let $J$ be a Markov chain with the two state space $E=\{1,2\}$ with intensity matrix

$$
\left(\begin{array}{ll}
q_{11} & q_{12} \\
q_{21} & q_{22}
\end{array}\right)=\left(\begin{array}{rr}
-1 & 1 \\
1 & -1
\end{array}\right) .
$$

Let $\lambda_{1}=1$ and $\lambda_{2}=2$, and let $G_{1}$ and $G_{2}$ be the exponential distributions with parameters 1 and 2 , respectively. Then the corresponding stationary distribution is $\left(\pi_{1}, \pi_{2}\right)=\left(\frac{1}{2}, \frac{1}{2}\right)$. Let $\kappa=4$ and $\eta=5$ be the relative safety loading for the insurer and the reinsurer, respectively. Finally, we assume that $b_{t} \geq \frac{1}{2}$. Then, for any $0<r<1$,

$$
r p_{\mathcal{R}}(t)-t \sup _{i=1,2} \lambda_{i} \int_{0}^{\infty}\left(\mathrm{e}^{r x}-1\right) G_{i}(\mathrm{~d} x) \geq \frac{r(1-2 r) t}{1-r} .
$$

Therefore, $R \geq \frac{1}{2}$, and corresponding ruin probability $\psi(x) \leq \mathrm{e}^{-x / 2}$.

\section{References}

[1] Asmussen, S. (2000). Ruin Probabilities. World Scientific, River Edge, NJ.

[2] Asmussen, S. And Kluppelberg, C. (1996). Large deviation results for subexponential tails, with applications to insurance risk. Stoch. Process Appl. 64, 103-125.

[3] BALdi, P. AND Piccioni, M. (1999). A representation formula for the large deviation rate function for the empirical law of a continuous time Markov chain. Statist. Prob. Lett. 41, 107-115. 
[4] Dembo, A. And Zeitouni, O. (1998). Large Deviation Technique and Applications, 2nd edn. Springer, New York.

[5] Deuschel, J. D. And Stroock, D. W. (1989). Large Deviations. Academic Press, Boston, MA.

[6] DJehiche, B. (1993). A large deviation estimate for ruin probabilities. Scand. Actuarial J. 1, 42-59.

[7] Djellout, H., Guillin, A. AND Wu, L. M. (1999). Large and moderate deviations for estimators of quadratic variational processes of diffusions. Statist. Inf. Stoch. Process. 2, 195-225.

[8] Duffield, N. G. ANd O'Connell, N. (1995). Large deviations and overflow probabilities for a single server queue, with applications. Math. Proc. Camb. Philos. Soc. 118, 363-374.

[9] Embrechts, P., Kluppelberg, C. And Mikosch, T. (1997). Modeling Extremal Events for Insurance and Finance. Springer, Berlin.

[10] Gao, F. Q. (1995). Small perturbation Cramer methods and moderate deviations for Markov processes. Acta Math. Sci. 15, 394-405.

[11] He, S. W., Wang, J. G. And Yan, J. A. (1992). Semimartingale Theory and Stochastic Calculus. Science Press, Beijing.

[12] KAAS, R. AND TANG, Q. (2005). A large deviation result for aggregate claim with dependent claims occurrences. Insurance Math. Econom. 36, 251-259.

[13] Macci, C. and Stabile, G. (2006). Large deviations for risk processes with reinsurance. J. Appl. Prob. 43, 713-728.

[14] Pham, H. (2007). Some applications and methods of large deviations in finance and insurance. In Paris-Princeton Lectures on Mathematical Finance (Lecture Notes Math. 1919), Springer, Berlin, pp. 191-244.

[15] Wu, L. M. (1995). Moderate deviations of dependent random variables related to CLT. Ann. Prob. 23, 420-445. 